\title{
Expression and Suppression of Human Telomerase RNA
}

\author{
S. Li AND E.H. BLACKBURN \\ Department of Biochemistry and Biophysics, University of California, San Francisco, California 94158-2517
}

\begin{abstract}
Telomeres are maintained by the ribonucleoprotein (RNP) enzyme telomerase, which replenishes telomeres through its unique mechanism of internal RNA-templated addition of telomeric DNA. Telomerase is active in most human cancers, typically because its core protein subunit, TERT, is up-regulated. Although the major known function of telomerase in cancer is to replenish telomeric DNA and maintain cell immortality, the regulation of the RNA component of telomerase is not well understood. In the course of investigations that have implicated telomerase RNA in key aspects of cancer progression, including metastasis, we explored some of the cis-acting elements affecting telomerase RNA expression and knockdown. The expression efficiency and subsequent RNA processing to produce the mature hTER differed considerably among various promoters. Together with other results, these findings establish that the crucial elements of the hTER gene affecting RNA-processing efficiency to produce the mature hTER RNA are the promoter and internal telomerase RNA-coding sequences.
\end{abstract}

\section{THE RNA COMPONENT OF TELOMERASE: AN UNUSUAL NONCODING RNA}

Elongation of human telomeres requires the enzymatic activity of the telomerase holoenzyme. In vitro, telomerase activity can be reconstituted by the catalytic subunit (hTERT) and the RNA subunit (called hTERC, hTER, or hTR). However, the routes of the RNA and protein components of telomerase from their generation to assembly into telomerase appear to be complex and likely to be highly regulated. The expression of hTERT is greatly diminished in most human adult somatic cells, with some exceptions, notably germ cells and stem cells. In contrast, the expression of hTER is readily detectable in a wide range of normal cells. The biological significance of this accumulation of telomerase RNA in human cells that lack significant levels of telomerase enzymatic activity is not well understood. Also still largely unknown are how the telomerase components are regulated, how the RNP telomerase enzyme complex is assembled, and how such assembly is controlled. We investigated the cis-acting elements of telomerase RNA gene constructs to explore how they affect the journey of telomerase RNA from gene to telomerase activity in human cells.

We analyzed expression and processing of human telomerase RNA in cultured transformed human cells, focusing on their dependence on cis-acting sequences in a variety of hTER gene constructs. Although this work was done in the course of experimenting with expression constructs designed with the goal of expressing human telomerase RNA efficiently in human tumor and other cells, the single-copy telomerase RNA gene is also an interesting small nuclear RNA (snRNA)-encoding gene for a number of reasons. First, in vertebrates, many small RNAs are encoded as multiple genes falling into gene families; single-copy snRNA genes are less common. Second, the transcriptional and processing machinery responsible for expression of telomerase RNA differs among different eukaryotic phyla. The transcription and expression of telomerase RNA have been characterized in diverse species, including ciliates, budding yeasts, and humans (Feng et al. 1995). It has been shown that ciliate telomerase RNA is transcribed by RNA polymerase III (pol III) (Yu et al. 1990; Romero and Blackburn 1991) and that the ciliate telomerase RNA promoter functionally resembles that of the vertebrate U6/7SK promoters, and also lacks cis-acting control elements that lie within the RNA-coding sequence. In this way, the ciliate telomerase RNA promoter is unlike the promoters of the 5S RNA and tRNA genes transcribed by RNA pol III (Romero and Blackburn 1991; Hargrove et al. 1999). In contrast to ciliates, in budding yeast and vertebrates, telomerase RNA expression is driven by an RNA pol II promoter. Third, the processing of telomerase RNA transcripts to yield the mature telomerase RNA competent for assembly into telomerase enzyme is not well understood. Ciliate telomerase RNA has a binding site for a telomerase-specific La family protein that is involved in its biogenesis and stability (Witkin and Collins 2004). In contrast, the mature processed form of budding yeast telomerase RNA contains a binding site for the Sm protein complex (Seto et al. 1999), which is involved in the biogenesis of spliceosomal snRNAs. In budding yeast, the RNA pol-II-transcribed telomerase RNA gene products include a polyadenylated transcript. Although normally present in very low amounts, in various mutants, this polyadenylated form of telomerase RNA accumulates relative to the mature RNA (Chapon et al. 1997). In contrast to the yeast telomerase RNA, studies of human telomerase RNA suggest that its transcript is nonpolyadenylated (Feng et al. 1995). Furthermore, in contrast to both ciliate and budding yeast telomerase RNAs, hTER contains a 3' nucleolar RNA-like box H/ACA domain (Mitchell et al. 1999). This box H/ACA domain is conserved among vertebrate telomerase RNAs, as is the presence of a small Cajal-body-specific RNA (scaRNA) sequence (Richard et al. 2003).

The detection of hTER in nucleoli (Wong et al. 2002) has further suggested that regulation of hTER biosynthesis and its incorporation into the telomerase RNP may be small nucleolar RNA (snoRNA)-like. Vertebrate 
snoRNAs are transcribed, and the mature snoRNA is processed, from a variety of genomic environments. The vertebrate snoRNAs that have been characterized the most extensively are encoded in intronic regions of snoRNA "host" genes, the transcripts of which often have a 5'-terminal oligopyrimidine sequence of unknown significance. Elements within the snoRNA-coding sequence itself direct snoRNA processing to release the mature snoRNA. In the case of the vertebrate box C/D snoRNAs, their processing occurs via exonucleolytic trimming, and interactions have been detected between the splicing of the host gene transcript and the snoRNA processing (Hirose and Steitz 2001; Hirose et al. 2003). A scaRNA sequence in an RNA directs that RNA to locate in Cajal bodies, nucleoplasmic bodies containing a variety of snRNAs and snoRNAs (Handwerger and Gall 2006). When its scaRNA sequence is mutated, hTER accumulates in the nucleolus (Richard et al. 2003). The expression of hTER thus may be regulated by a transcription complex characteristic of an snRNA or snoRNA promoter (Feng et al. 1995; Richard et al. 2003).

Recent work (see Kiss et al., Terns and Terns, both this volume) has identified certain Cajal body proteins that are specifically associated with hTER, as well as nucleolar proteins (Jady et al. 2004; Zhu et al. 2004). In cells lacking substantial telomerase enzymatic activity, the telomerase RNA that can accumulate in such cells is presumably stable enough to accumulate in the absence of expressed TERT protein, and potential candidates for its binding partners in these cells include the Cajal body proteins and the dyskerin complex (DKC) (Vulliamy et al. 2001; Hamma et al. 2005). Cytological analyses of telomerase components by methods that can detect labeled hTER or hTERT in cells suggest dynamic movement of telomerase RNA between different cellular compartments, including the nucleus and nucleolus (Jady et al. 2006; Tomlinson et al. 2006).

Understanding where hTER is located in its different cellular milieus, and what affects its maturation and stability, are important in order to experimentally manipulate human telomerase RNA. Therefore, we first examined the expression of human telomerase RNA from expression cassettes with various upstream and downstream sequences flanking the 451-nucleotide-long coding sequence of the mature hTER. In addition, we investigated some of the expression characteristics of ribozymes and small hairpin interfering RNA (shRNAi) constructs that affect their ability to knock down human telomerase RNA.

\section{UPSTREAM, BUT NOT DOWNSTREAM, GENE SEQUENCES AFFECT THE EXPRESSION AND PROCESSING OF TELOMERASE RNA}

We analyzed a set of hTER constructs engineered to test their utility for hTER expression in human cancer cells. The findings uncovered a number of interesting features of the telomerase RNA gene. These features are reminiscent of the expression control of other box H/ACA RNAs of vertebrates (Wang and Meier 2004).

Previous data suggested that the total amount of hTER accumulation in human tumor cells is regulated, since only low levels of hTER accumulated even when ectopically expressed from strong promoters in the context of an adenoviral, plasmid, or retroviral vector (Marusic et al. 1997; Kim et al. 2001). Furthermore, in previous work, when hTER gene transcripts were generated from retroviral or plasmid-borne cytomegalovirus (CMV) promoterdriven constructs, most of the transcript was apparently unprocessed, since the predominant product observed in tumor cell lines was the polyadenylated form of the transcript that had been produced using the polyadenylation site engineered into the construct (typically, the retroviral polyadenylation site or a viral SV40 polyadenylation site) (Kim et al. 2001). Previous data have indicated that such polyadenylated hTER transcripts are not assembled into the telomerase RNP (Feng et al. 1995; Mitchell et al. 1999). Therefore, first, we sought to identify an ideal promoter for telomerase RNA expression and correct processing by testing a variety of RNA pol II and RNA pol III promoters. The RNA pol II promoters tested were the CMV (viral), CAG, PGK (cellular protein-coding genes), and IU1 promoters. The IU1 promoter was made from one of the gene family members of the U1 snRNA gene family. U6 and tRNA RNA gene promoters were the pol III promoters chosen for testing (Fig. 1). Second, to determine the effects of downstream sequences on expression and processing efficiency, transcription termination sequences unique for IU1, U6, tRNA, and CMV were used in the expression cassettes as described previously (Bertrand et al. 1997). Flanked by these $5^{\prime}$ and $3^{\prime}$ sequences, two types of hTER gene constructs were tested: an hTER DNA fragment containing only the sequence of the mature 451-nucleotide hTER (nucleotides 1-451) and a 598-nucleotide long sequence including the 451-nucleotide hTER sequence plus the flanking 137 nucleotides of the 3' genomic sequence. Each of these hTER expression cassettes was engineered into a lentiviral expression vector, which was chosen for its high transduction capability and the stable and efficient expression of genes from the genomically integrated, proviral form of the lentivector-borne genes.

To monitor the expression of hTER under different promoters, we utilized the "VA13" cell line WI-38 VA13/2RA (SV40-immortalized WI-38). This SV40transformed human fibroblast line has previously been shown to have no endogenous hTER or hTERT expression (Bryan et al. 1997). Addition of constructs expressing both these genes, hTERT and hTER, reconstitutes activity in these cells (Bryan et al. 1997). Thus, VA13 cells have served as a useful "blank slate" on which to build and study telomerase activity with engineered components in the context of a cell. We have previously shown that expression of hTER can result in stable accumulation of the hTER RNA at levels that are unchanged even when hTERT is co-overexpressed in the same cells (Li et al. 2004). Hence, the stability of hTER in VA13 cells is not dependent on the level of hTERT protein. The lentivirus carrying different hTER expression cassettes also carried a green fluorescent protein (GFP) expression element to monitor infection efficiencies (which typically produced more than $90 \%$ of the cells that were GFP-positive). The VA13 cells were infected with each lentiviral 
A
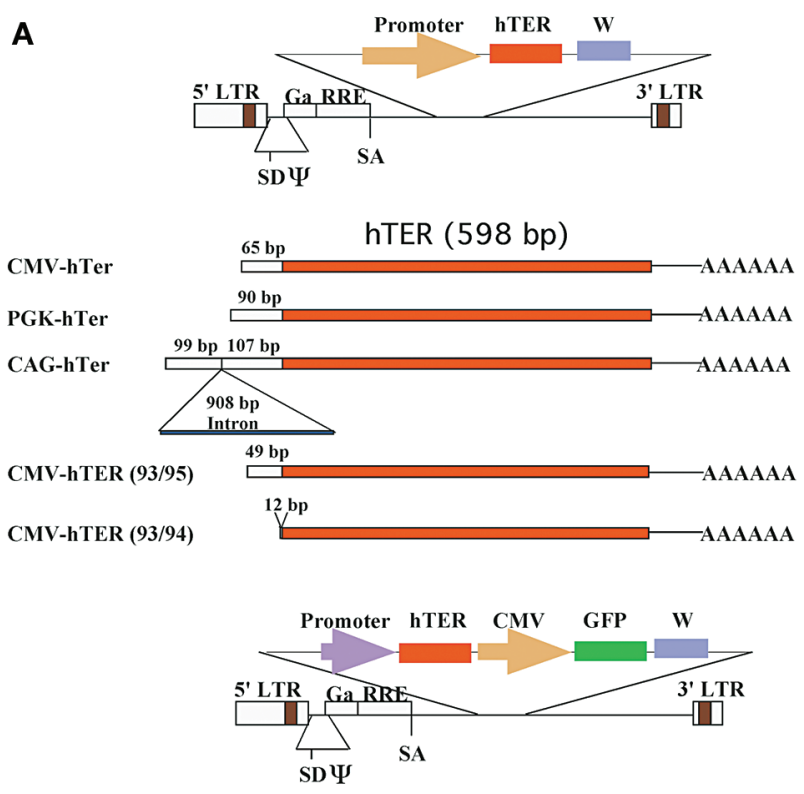

IU1-hTER

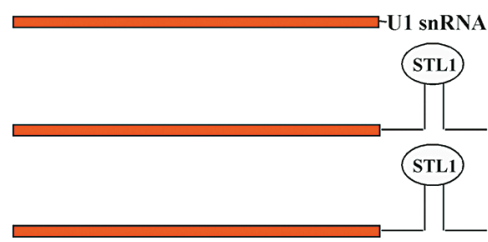

U6-hTER

tRNA-hTER
B

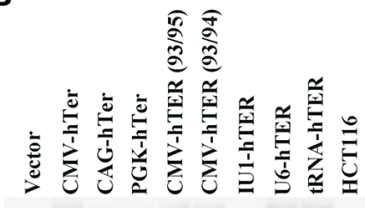

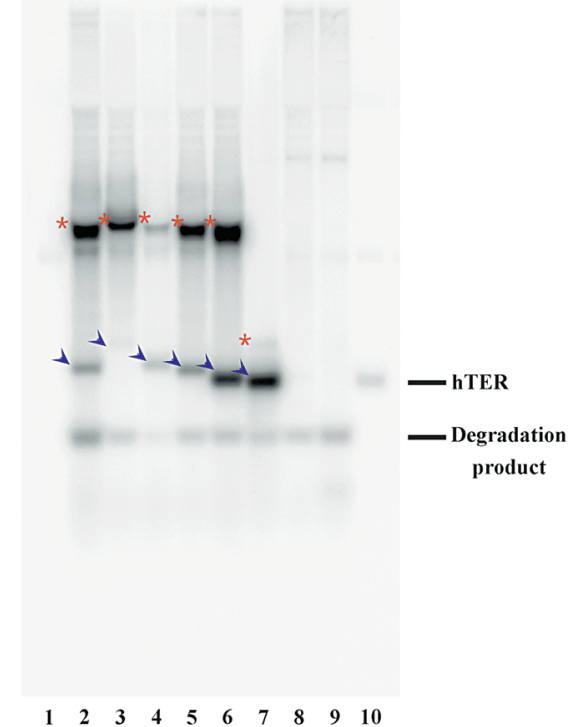

Figure 1. Expression of human telomerase RNA from lentiviral constructs with various promoters. $(A)$ Lentiviral vectors: Only the relevant portion of the plasmid is illustrated. The hTER expression cassettes inserted into the lentivector were driven by various promoters as indicated. For the expression construct using the IU1, U6, and tRNA promoters, a GFP reporter expression cassette was also engineered to monitor the transduction efficiency. (W) Woodchuck hepatitis virus posttranslational regulatory element (Zufferey et al. 1999) placed at the $3^{\prime}$ end of GFP to enhance GFP expression. Schematic of hTER transcript from various expression cassettes: The 598-bp hTER fragment is shown (red rectangle). The additional sequence $5^{\prime}$ to hTER (white rectangle) is vector-derived sequence which follows the transcription initiation site. Note that the $3^{\prime}$ termination sequence is different due to the nature of different expression cassettes. Although a transcriptional termination site is found immediately downstream from the IU1-hTER cassette that is also utilized by RNA pol II, this termination site does not appear to cause early termination of the full-length lentiviral transcript, as a hightiter recombinant viral supernatant can be produced using these lentivectors. In contrast, when a polyadenylation signal is inserted into the viral genome in a forward orientation, the viral titer is dramatically reduced due to loss of full-length viral RNA transcript (Coffin et al. 1997). (B) Northern blotting analysis of telomerase RNA expressed from different promoters in VA13 cells 4 days after lentiviral infection. (Lane 1) Control virus; (lane 2) CMV-promoter-driven hTER; (lane 3) CAG promoter-driven hTER; (lane 4) PGK-promoter-driven hTER; (lane 5) CMV-promoter-driven hTER with shorter 5' additional sequence; (lane 6) CMV-promoter-driven hTER with shortest 5' additional sequence; (lane 7) IU1-promoter-driven hTER (598) and GFP reporter; (lane 8) U6-promoter-driven hTER and GFP reporter; (lane 9) tRNA-promoter-driven hTER and GFP reporter; (lane 10) RNA from HCT116 colon cancer cells. The asterisks indicate unprocessed hTER transcripts. The arrowheads indicate mature telomerase RNA transcripts.

construct, the unabsorbed virus was washed out of the medium after 8 hours, and at 4 days postinfection of the virus, total cellular RNA was prepared as described previously (Li et al. 2004). The expression of hTER was then detected and analyzed by northern hybridization.

Each of these RNA pol II promoter-derived expression cassettes resulted in readily detected expression of hTER, although the expression efficiency and subsequent RNA processing to produce the mature hTER differed considerably among the promoters. When hTER was expressed from the IU1 RNA pol II promoter, all of the transcript was correctly processed at the $3^{\prime}$ end. In contrast to hTER expressed from the IU1 promoter, the majority of hTER transcripts expressed from the CMV, CAG, and PGK promoters are polyadenylated, and only a small portion of the hTER transcript is properly processed to the 451-base mature form. Similar results were observed previously for the CMV promoters in adenoviral plasmids and retroviral vectors (Marusic et al. 1997; Kim et al. 2001). These data suggested that transcription which directs the hTER transcript into the polyadenylation pathway may inhibit the maturation of hTER in vivo. In addition, as reported previously, the expression of endogenous and ectopically expressed WT-hTER in two human tumor cell lines - the melanoma line LOX and the bladder cancer line UM-UC3 cells - has been analyzed by northern hybridization ( $\mathrm{Li}$ et al. 2004). Overexpression of this ectopically expressed WT-hTER was observed in both cell lines, suggesting that expression of telomerase RNA in human tumor cell lines using the IU1 promoter may bypass the regulation of total hTER accumulation. Such regulation had been suggested from previous studies in the human prostate cancer cell line LNCaP using an expression cassette with the viral CMV promoter and an SV40 polyadenylation site (Kim et 
al. 2001). In contrast to the pol II promoters, no hTER transcript was detectable when the U6 or tRNA promoter was used, despite delivery of the expression cassettes to more than $95 \%$ of cells, as indicated by their GFP positivity (data not shown). This result suggested that the hTER transcript derived from RNA pol III may not be stable, since the same promoter yields robust expression of ribozyme transcripts (Bertrand et al. 1997).

In addition, the dependence of the efficiency of 3 -end processing on the sequence at the $5^{\prime}$ end of the transcript was tested. We kept the CMV promoter the same but varied the $5^{\prime}$-end sequence of the transcript. As shown in Figure 1 (lanes 2, 5, and 6), although the CMV construct with a 12-base insertion at the $5^{\prime}$ end of the hTER-coding sequence was expressed at higher overall levels than that with a 49-base or 65-base insert, the ratio of the unprocessed full-length polyadenylated transcript to the processed mature-length hTER was not significantly altered (Fig. 1, lanes 2, 5, and 6). Therefore, the $5^{\prime}$-end sequence of the transcript did not affect the relative degrees of processing of the transcripts via polyadenylation versus $3^{\prime}$ end processing to form mature hTER. We next tested whether an intron in the transcript could improve the ratio of processed to unprocessed hTER transcript. The presence of an intron affects the processing pathway of mRNA transcripts; however, the CAG promoter construct did not result in high-level production to mature hTER (Fig. 1, lane 3). These findings suggest that the primary $5^{\prime}$ determinant of relative processing of a transcript into mature hTER is the promoter, and not sequences within or downstream from hTER.

As previously reported (Li et al. 2004), two different hTER-containing fragments were engineered to be expressed from the IU1 promoter: The first one, IU1-hTER (598), contains mature hTER (nucleotides 1-451) and the flanking 137 nucleotides of $3^{\prime}$ genomic sequence; the second one, IU1-hTER (451), contains only nucleotides 1-451 of the mature hTER. Expression levels of both hTER gene constructs were similar, and only the transcript corresponding to the mature hTER was observed in both cases (Li et al. 2004). To test whether hTER expressed from the IU1 promoter can be assembled into functional telomerase enzyme, in vitro TRAP (telomere repeat amplification protocol) assays for telomerase enzymatic activity were conducted on cell lysates from VA13 (hTert) cells infected with both of these types of hTER expression cassettes (598 or 451). Typical telomerase product ladders were detectable only in cell extracts expressing both hTERT and hTER. The results were similar with either the IU1-hTER (598) or IU1-hTER (451) expression cassette. Nucleotides 1-451, corresponding to the full-length hTER, thus appear to comprise sufficient RNA transcript information to direct hTER maturation and incorporation into a functional telomerase RNP enzyme complex (Li et al. 2004).

Finally, we also tested cassettes in which the 3 '-flanking sequence immediately downstream from the coding sequence was a different sequence, unrelated to the natural hTER-flanking sequence that was used in the constructs listed above. There was no effect on the processing efficiency of varying this downstream sequence (results not shown). Thus, these results are consistent with the conclusion that the processing of the hTER transcript to produce the $3^{\prime}$ end of the mature hTER is controlled by sequences in the coding region, consistent with the results for other vertebrate snoRNAs of the box H/ACA class (Wang and Meier 2004; Hamma et al. 2005).

A characteristic partial-length fragment of hTER was commonly seen in these ectopically expressed hTER transcripts. This fragment was mapped and found to consist of a $3^{\prime}$-end fragment of hTER, from which the $5^{\prime}$ portion of the telomerase RNA, consisting of the universally conserved core of the telomerase RNA that contains the templating domain and conserved TER pseudoknot, has been removed. This $5^{\prime}$ half of the hTER is apparently readily degraded, as it is detected only variably, and as an indistinct smear of fragments, in these experiments, leaving only the more stable $3^{\prime}$ portion of the telomerase RNA. This remaining $3^{\prime}$-half fragment of hTER contains the previously identified conserved regions 4-7 of vertebrate telomerase RNA (Chen et al. 2000), which include the box H/ACA structure. Since box H/ACA snoRNAs bind the four-protein complex DKC core complex (Vulliamy et al. 2001; Hamma et al. 2005), this complex likely stabilizes this hTER fragment. A similar 3'-half degradation fragment was characterized in $\mathrm{LNCaP}$ human prostate cancer cells (Kim et al. 2001; and M. Kim and E.H. Blackburn, unpubl.). It is unknown whether this protected 3 '-half fragment results from an endonucleolytic cleavage event that cuts off the universal core region, or from $5^{\prime}$ to $3^{\prime}$ exonuclease action that reaches a stopping point between the telomerase RNA universal conserved domain and the box H/ACA domain, or from combined endo- and exonucleolytic activities. Whether this consistently observed $3^{\prime}$-half fragment of hTER is a normal intermediate in a breakdown pathway of the endogenous telomerase RNA or has any other function is not known.

\section{PROMOTERS DRIVING THE EXPRESSION OF RIBOZYMES OR SIRNAS AFFECT THEIR EFFICIENCY IN KNOCKING DOWN HUMAN TELOMERASE RNA}

For experimental purposes, we wanted to knock down telomerase RNA in human cells ( $\mathrm{Li}$ et al. 2004). Therefore, we examined features of ribozymes or small interfering RNA gene constructs designed with the goal of optimal knockdown of hTER. A priori, the RNA knockdown agent must encounter its target RNA in the cell. However, unlike most RNAs that are commonly targeted by RNAi pathways, both experimentally and in natural settings, hTER is a noncoding, non-mRNA target. Furthermore, in contrast to the typical mRNA targets of RNA knockdown, generating the functional form of telomerase RNA in human telomerase does not appear to involve processing through the polyadenylation pathway. As described above, it is not known whether endogenous hTER is naturally subject to regulation via RNAi or by microRNA pathways. We have previously reported that the mouse telomerase RNA in a mouse melanoma cell line can be knocked down to approximately $20-30 \%$ of its normal level by hammerhead ribozyme constructs, with the ribozyme expression driven from a CMV promoter 
(Nosrati et al. 2004). Similar results were observed in human cancer cell lines when ribozymes targeting human telomerase RNA were expressed from the U3B7 promoter (Bertrand et al. 1997) (data not shown). Interestingly, a similar level of knockdown was observed in these human cells regardless of whether these ribozymes were expressed from a tRNA, U6, or U3B7 promoter, even though each promoter is predicted to result in its transcript accumulating in different cellular compartments (Bertrand et al. 1997). In addition, we tested different shRNA constructs for their efficacy in knocking down the levels of hTER and telomerase activity. The shRNAs were expressed from various expression cassettes in lentiviral constructs. We found that hTER can be efficiently knocked down by an shRNA expressed from a U6 promoter ( $\mathrm{Li}$ et al. 2004, 2005). These limited findings provisionally suggest that cellular localization of the ribozyme or shRNA transcripts may not be a major determinant of their effectiveness in hTER knock-down. In summary, telomerase RNA expression and function require trafficking within the cell via specific pathways, and further studies will be needed for a fuller understanding of the biogenesis of this RNA.

\section{ACKNOWLEDGMENTS}

The authors gratefully acknowledge support for this work provided by the UCSF Comprehensive Cancer Center Breast Cancer SPORE CA58207, grant CA96840 from the National Cancer Institute, and the Bernard Osher Foundation (to E.H.B.), and support from the Damon Runyon Cancer Research Foundation (to S.L.).

\section{REFERENCES}

Bertrand E., Castanotto D., Zhou C., Carbonnelle C., Lee N.S., Good P., Chatterjee S., Grange T., Pictet R., Kohn D., et al. 1997. The expression cassette determines the functional activity of ribozymes in mammalian cells by controlling their intracellular localization. RNA 3: 75 .

Bryan T.M., Marusic L., Bacchetti S., Namba M., and Reddel R.R. 1997. The telomere lengthening mechanism in telomerase-negative immortal human cells does not involve the telomerase RNA subunit. Hum. Mol. Genet. 6: 921.

Chapon C., Cech T.R., and Zaug A.J. 1997. Polyadenylation of telomerase RNA in budding yeast. RNA 3: 1337.

Chen J.L., Blasco M.A., and Greider C.W. 2000. Secondary structure of vertebrate telomerase RNA. Cell 100: 503.

Coffin J.M., Hughes S.H., and Varmus H.E., eds. 1997. Retroviruses. Cold Spring Harbor Laboratory Press, Cold Spring Harbor, New York.

Feng J., Funk W.D., Wang S.S., Weinrich S.L., Avilion A.A., Chiu C.P., Adams R.R., Chang E., Allsopp R.C., Yu J., et al. 1995. The RNA component of human telomerase. Science 269: 1236.

Hamma T., Reichow S.L., Varani G., and Ferre-D'Amare A.R. 2005. The Cbf5-Nop10 complex is a molecular bracket that organizes box H/ACA RNPs. Nat. Struct. Mol. Biol. 12: 1101.

Handwerger K.E. and Gall J.G. 2006. Subnuclear organelles: New insights into form and function. Trends Cell Biol. 16: 19.

Hargrove B.W., Bhattacharyya A., Domitrovich A.M., Kapler G.M., Kirk K., Shippen D.E., and Kunkel G.R. 1999. Identification of an essential proximal sequence element in the promoter of the telomerase RNA gene of Tetrahymena thermophila. Nucleic Acids Res. 27: 4269.

Hirose T. and Steitz J.A. 2001. Position within the host intron is critical for efficient processing of box C/D snoRNAs in mammalian cells. Proc. Natl. Acad. Sci. 98: 12914.

Hirose T., Shu M.D., and Steitz J.A. 2003. Splicing-dependent and -independent modes of assembly for intron-encoded box C/D snoRNPs in mammalian cells. Mol. Cell 12: 113.

Jady B.E., Bertrand E., and Kiss T. 2004. Human telomerase RNA and box H/ACA scaRNAs share a common Cajal bodyspecific localization signal. J. Cell Biol. 164: 647.

Jady B.E., Richard P., Bertrand E., and Kiss T. 2006. Cell cycledependent recruitment of telomerase RNA and Cajal bodies to human telomeres. Mol. Biol. Cell 17: 944.

Kim M.M., Rivera M.A., Botchkina I.L., Shalaby R., Thor A., and Blackburn E.H. 2001. A low threshold level of expression of mutant-template telomerase RNA is sufficient to inhibit human tumor cell growth. Proc. Natl. Acad. Sci. 98: 7982.

Li S., Crothers J., Haqq C.M., and Blackburn E.H. 2005. Cellular and gene expression responses involved in the rapid growth inhibition of human cancer cells by RNA interference-mediated depletion of telomerase RNA. J. Biol. Chem. 280: 23709.

Li S., Rosenberg J.E., Donjacour A.A., Botchkina I.L., Hom Y.K., Cunha G.R., and Blackburn E.H. 2004. Rapid inhibition of cancer cell growth induced by lentiviral delivery and expression of mutant-template telomerase RNA and antitelomerase short-interfering RNA. Cancer Res. 64: 4833.

Marusic L., Anton M., Tidy A., Wang P., Villeponteau B., and Bacchetti S. 1997. Reprogramming of telomerase by expression of mutant telomerase RNA template in human cells leads to altered telomeres that correlate with reduced cell viability. Mol. Cell. Biol. 17: 6394.

Mitchell J.R., Cheng J., and Collins, K. 1999. A box H/ACA small nucleolar RNA-like domain at the human telomerase RNA 3' end. Mol. Cell. Biol. 19: 567.

Nosrati M., Li S., Bagheri S., Ginzinger D., Blackburn E.H., Debs R.J., and Kashani-Sabet M. 2004. Antitumor activity of systemically delivered ribozymes targeting murine telomerase RNA. Clin. Cancer Res. 10: 4983.

Richard P., Darzacq X., Bertrand E., Jady B.E., Verheggen C., and Kiss T. 2003. A common sequence motif determines the Cajal body-specific localization of box H/ACA scaRNAs. EMBO J. 22: 4283.

Romero D.P. and Blackburn E.H. 1991. A conserved secondary structure for telomerase RNA. Cell 67: 343.

Seto A.G., Zaug A.J., Sobel S.G., Wolin S.L., and Cech T.R. 1999. Saccharomyces cerevisiae telomerase is an Sm small nuclear ribonucleoprotein particle. Nature 401: 177.

Tomlinson R.L., Ziegler T.D., Supakorndej T., Terns R.M., and Terns M.P. 2006. Cell cycle-regulated trafficking of human telomerase to telomeres. Mol. Biol. Cell 17: 955.

Vulliamy T., Marrone A., Goldman F., Dearlove A., Bessler M., Mason P.J., and Dokal I. 2001. The RNA component of telomerase is mutated in autosomal dominant dyskeratosis congenita. Nature 413: 432.

Wang C. and Meier U.T. 2004. Architecture and assembly of mammalian H/ACA small nucleolar and telomerase ribonucleoproteins. EMBO J. 23: 1857.

Witkin K.L. and Collins K. 2004. Holoenzyme proteins required for the physiological assembly and activity of telomerase. Genes Dev. 18: 1107.

Wong J.M., Kusdra L., and Collins K. 2002. Subnuclear shuttling of human telomerase induced by transformation and DNA damage. Nat. Cell Biol. 4: 731.

Yu G.L., Bradley J.D., Attardi L.D., and Blackburn E.H. 1990. In vivo alteration of telomere sequences and senescence caused by mutated Tetrahymena telomerase RNAs. Nature 344: 126

Zhu Y., Tomlinson R.L., Lukowiak A.A., Terns R.M., and Terns M.P. 2004. Telomerase RNA accumulates in Cajal bodies in human cancer cells. Mol. Biol. Cell 15: 81.

Zufferey R., Donello J.E., Trono D., and Hope T.J. 1999. Woodchuck hepatitis virus posttranscriptional regulatory element enhances expression of transgenes delivered by retroviral vectors. $J$. Virol. 73: 2886. 


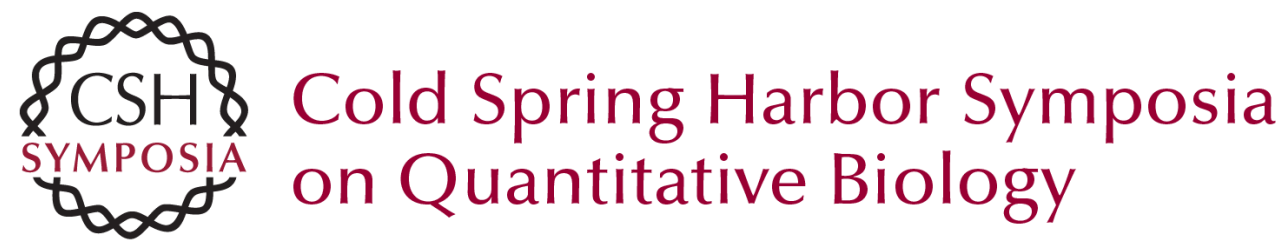

\section{Expression and Suppression of Human Telomerase RNA}

S. LI and E.H. BLACKBURN

Cold Spring Harb Symp Quant Biol 2006 71: 211-215

Access the most recent version at doi:10.1101/sqb.2006.71.009

References This article cites 29 articles, 17 of which can be accessed free at: http://symposium.cshlp.org/content/71/211.full.html\#ref-list-1

\section{License}

Email Alerting Receive free email alerts when new articles cite this article - sign up in Service the box at the top right corner of the article or click here. 